\title{
The Drinfel'd Twisted XYZ Model
}

\section{Rubik Poghossian *}

\author{
Address \\ Nussallee 12, Physikalisches Institut der Universität Bonn, 53115 Bonn \\ E-mail: 'poghos@th.physik. uni-bonn.de'
}

AbSTRACT: We construct a factorizing Drinfel'd twist for a face type model equivalent to the $X Y Z$ model. Completely symmetric expressions for the operators of the monodromy matrix are obtained

\section{Introduction}

Several integrable quantum spin chain models within the range of the algebraic Bethe ansatz method have a distinguished basis of states, which minimizes quantum effects. That is, the quasiparticle creation and annihilation operators in this basis have an appearance devoid of polarization clouds. For the $X X X$ and $X X Z$ models with underlying group $s l(2)$ the bases in question were found by Maillet and Sanchez de Santos [1] through the construction of a generalized Drinfeld twist $[\overline{2}]$. The ensuing representation of the quantum monodromy matrices coincides, as noted by Terras [3], for the case of the rational $X X X$ model with the representation provided by Sklyanin's functional Bethe ansatz method An obvious generalization of Sklyanin's method (substituting polynomials in the spectral parameter by polynomials in the exponential of the spectral parameter) leads us to the conclusion that an analogous coincidence holds true for the trigonometric $X X Z$ model. The purpose of this talk (based on the $\overline{\overline{7}} \mid \bar{l}$ ) is to report on the generalization of the above results to the elliptic $s l(2)$ $X Y Z$ model. For this sake we make use of Baxter's map of the $X Y Z$ model onto an ice type model [5]. This brings us formally near to the $X X X$ and $X X Z$ models and allows us to construct the corresponding $\mathrm{F}$-transformation in the quantum space and write down explicit expres-

*In collaboration with T.-D.Albert, H.Boss, R.Flume, K.Ruhlig sions for the monodromy matrix elements in this new basis

\section{2. $X Y Z$ model and its relation to ice- type models}

In the framework of the Algebraic Bethe Ansatz [8, ] solution of the Yang-Baxter equation

$$
\begin{array}{r}
R_{12}\left(\lambda_{1}-\lambda_{2}\right) R_{13}\left(\lambda_{1}-\lambda_{3}\right) R_{23}\left(\lambda_{2}-\lambda_{3}\right)= \\
\quad R_{23}\left(\lambda_{2}-\lambda_{3}\right) R_{13}\left(\lambda_{1}-\lambda_{3}\right) R_{12}\left(\lambda_{1}-\lambda_{2}\right)
\end{array}
$$

with

$$
R^{x y z}(\lambda-\mu)=\left(\begin{array}{cccc}
a & 0 & 0 & d \\
0 & b & c & 0 \\
0 & c & b & 0 \\
d & 0 & 0 & a
\end{array}\right)
$$

where

$$
\begin{aligned}
& a=\frac{\Theta(2 \eta) \Theta(\lambda-\mu)}{\Theta(0) \Theta(\lambda-\mu+2 \eta)}, \\
& b=\frac{\Theta(2 \eta) H(\lambda-\mu)}{\Theta(0) H(\lambda-\mu+2 \eta),} \\
& c=\frac{H(2 \eta) \Theta(\lambda-\mu)}{\Theta(0) H(\lambda-\mu+2 \eta)}, \\
& d=\frac{H(2 \eta) H(\lambda-\mu)}{\Theta(0) \Theta(\lambda-\mu+2 \eta)}
\end{aligned}
$$

with the notation $H(u)=\vartheta_{1}\left(\frac{u}{2 K}, q\right), \Theta(u)=$ $\vartheta_{4}\left(\frac{u}{2 K}, q\right)$ and $\vartheta_{4}(z, q)=\sum_{m \in Z}(-1)^{n} q^{n^{2}} e^{2 \pi i n z}$, 
$\vartheta_{1}(z, q)=-i q^{\frac{1}{4}} e^{i \pi z} \theta_{4}(z+\tau / 2, q)$ are the standard theta-functions of a single complex variable. The somewhat different parametrization as compared to [i, is due to the normalization in order to achieve unitarity of the R-matrix.

The monodromy matrix $T\left(\lambda,\left\{\lambda_{i}\right\}\right)$ (generalized to the inhomogeneous chain [0. $\left[\begin{array}{l}0 \\ 0\end{array}\right.$ given as the ordered product of Lax operators $L_{i}\left(\lambda-\lambda_{i}\right)=R_{0 i}\left(\lambda-\lambda_{i}\right)$

$$
\begin{array}{r}
T\left(\lambda,\left\{\lambda_{i}\right\}\right)= \\
L_{N}\left(\lambda-z_{N}\right) \ldots L_{2}\left(\lambda-z_{2}\right) L_{1}\left(\lambda-z_{1}\right)= \\
\left(\begin{array}{ll}
A\left(\lambda,\left\{\lambda_{i}\right\}\right) & B\left(\lambda,\left\{\lambda_{i}\right\}\right) \\
C\left(\lambda,\left\{\lambda_{i}\right\}\right) & D\left(\lambda,\left\{\lambda_{i}\right\}\right)
\end{array}\right) .
\end{array}
$$

The presence of the Boltzmann weight $d$ in Eq. (2.2) reflects the non-conservation of spin, which is responsible for the absence of a local vacuum for the Lax operator associated with the above R-matrix.

To circumvent the problems arising from the eight vertex nature, we use the vertex-face map established by Baxter [5] $[\overline{1}$ ] to obtain a $X X Z$ type (six vertex) $R$-matrix by exploiting the relation

$$
\begin{gathered}
R^{x y z}(\lambda-\mu) \phi_{l, l^{\prime}} \otimes z_{m^{\prime}, l^{\prime}}= \\
\sum_{m} w\left(m, m^{\prime} \mid l, l^{\prime}\right) \phi_{m, m^{\prime}} \otimes z_{m, l}
\end{gathered}
$$

valid for all integers $l, l^{\prime}, m, m^{\prime}$ such that $\mid l-$ $l^{\prime}|=| l^{\prime}-m^{\prime} \mid=1$ and the summation on the r.h.s. is over integers $m$ s.t. $\left|m-m^{\prime}\right|=|m-l|=1$.

The two dimensional vectors $\phi, z$ are given by

$$
\begin{aligned}
& \phi_{l, l+1}=X\left(s_{l}+\mu\right) ; z_{l+1, l}=X\left(s_{l}+\lambda\right) \\
& \phi_{l+1, l}=X\left(t_{l+1}-\mu\right) ; z_{l-1, l}=X\left(t_{l}-\lambda\right)
\end{aligned}
$$

with $X(u)=\left(\begin{array}{c}H(u) \\ \Theta(u)\end{array}\right)$ and the abbrevation $s_{l}=s+$ $2 \eta l$, where $s, t$ are arbitrary complex parameters.

The Boltzmann weights are given by $(h(u)$ $\left.=H(u) \Theta(u) ; \omega_{l}=\left(\frac{s+t}{2}+2 \eta l-K\right)\right)$ :

$$
\begin{array}{r}
a_{l}=a_{l}^{\prime}=1 ; \\
b_{l}=\frac{h(\lambda) h\left(\omega_{l-1}\right)}{h(\lambda+2 \eta) h\left(\omega_{l}\right)} ; \\
b_{l}^{\prime}=\frac{h(\lambda) h\left(\omega_{l+1}\right)}{h(\lambda+2 \eta) h\left(\omega_{l}\right)} ;
\end{array}
$$

$$
\begin{gathered}
c_{l}=\frac{h(2 \eta) h\left(\omega_{l}-\lambda\right)}{h(\lambda+2 \eta) h\left(\omega_{l}\right)} ; \\
c_{l}^{\prime}=\frac{h(2 \eta) h\left(\omega_{l}+\lambda\right)}{h(\lambda+2 \eta) h\left(\omega_{l}\right)},
\end{gathered}
$$

where we have introduced the notations: $a_{l}=$ $w(l-1, l \mid l-2, l-1), b_{l}=w(l+1, l \mid l, l-1)$, $c_{l}=w(l+1, l \mid l, l+1), a_{l}^{\prime}=w(l+1, l \mid l+2, l+1)$, $b_{l}^{\prime}=w(l-1, l \mid l, l+1), c_{l}^{\prime}=w(l-1, l \mid l, l-1)$. These weights can be arranged into a matrix

$$
R_{12}(l)=\left(\begin{array}{cccc}
a_{l} & 0 & 0 & 0 \\
0 & b_{l} & c_{l} & 0 \\
0 & c_{l}^{\prime} & b_{l}^{\prime} & 0 \\
0 & 0 & 0 & a_{l}^{\prime}
\end{array}\right)
$$

which fulfills the modified Yang-Baxter equation $[1 \overline{1} \overline{1}],[1 \overline{2} \overline{1}]$

$$
\begin{array}{r}
R_{12}\left(l-\sigma_{3}\right) R_{13}(l) R_{23}\left(l-\sigma_{1}\right)= \\
R_{23}(l) R_{13}\left(l-\sigma_{2}\right) R_{12}(l) .
\end{array}
$$

The monodromy matrix related to this modified Yang-baxter equation is

$$
\begin{array}{r}
T_{0,1 \ldots N}(l)= \\
R_{0 N}\left(l-\sigma_{1}-\ldots-\sigma_{N-1}\right) \ldots R_{02}\left(l-\sigma_{1}\right) R_{01}(l),
\end{array}
$$

where 0 denotes the horizontal auxiliary space (with the asssociated spectral parameter $\lambda_{0}$ ), the positive integers $1, \ldots, N$ label the vertical local quantum spaces which span the physical Hilbert space $\mathcal{H}_{N}$ (with associated local inhomogeneities $\left.\left\{\lambda_{i}\right\}\right)$, and $\sigma_{i}$ equals \pm 1 depending on whether the arrow in the $i$-th space is up or down (right/ left for the horizontal space). It also sets our convention to associate the integer in the right lower corner of the graphical representation of the monodromy matrix. From (2.9i) follows the equation for the monodromy matrices

$$
\begin{array}{r}
R_{00^{\prime}}\left(l-\sigma_{1}-\ldots-\sigma_{N}\right) T_{0}(l) \otimes T_{0^{\prime}}\left(l-\sigma_{0}\right)= \\
T_{0^{\prime}}(l) \otimes T_{0}\left(l-\sigma_{0^{\prime}}\right) R_{00^{\prime}}(l) .
\end{array}
$$

It is easy to check that the unitarity relation $R_{21} R_{12}=\mathbb{I}$ is satisfied too.

The construction of the eigenvalues of the transfer matrix obtained from the initial monodromy matrix (2.4) using the modified monodromy matrix $\left({ }^{2} .10^{\prime}\right)$ is explained in reference 
[1] (One has however to keep in mind that our

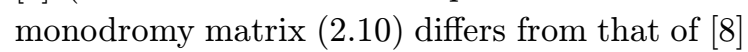
by an additional change of basis in the quantum space).

We will concentrate in what follows on the computation of a factorizing $F$-matrix for the monodromy matrix $\left({ }^{2} \cdot \overline{1} \cdot \overline{1}\right)$.

\section{The $F$ basis}

The factorizing $F$-matrix for two sites defined by the relation $F_{21} R_{12}=F_{12}$ is

$$
F_{12}=\left(\begin{array}{cccc}
1 & 0 & 0 & 0 \\
0 & 1 & 0 & 0 \\
0 & c^{\prime} & b^{\prime} & 0 \\
0 & 0 & 0 & 1
\end{array}\right)_{[12]} .
$$

The proof of the factorization property amounts to checking the same relations as those in the proof of the unitarity of the R-matrix above.

The factorizing $F$-matrix for $N$ sites ( $N$ quantum spaces) turns out to be given by formally the same expression as found in [6] for the $X X X$ model

$$
\begin{aligned}
F_{1 \ldots N}(l) & =\sum_{\alpha \in Z_{2}^{N}} P_{\alpha} R_{1 \ldots N}^{\sigma_{\alpha}}(l)\left(z_{1}, \ldots, z_{N}\right) \\
P_{\alpha} & =\prod_{i=1}^{N} P_{i}^{\alpha_{i}},
\end{aligned}
$$

where $P_{i}^{\alpha_{i}}$ projects on the $\alpha_{i}$-th component in the $i$-th space and the permutation $\sigma_{\alpha}$ is uniquely determined through the conditions

$$
\begin{array}{ll}
\alpha_{\sigma_{\alpha}(i+1)} \geq \alpha_{\sigma_{\alpha}(i)} \quad \text { if } \quad \sigma_{\alpha}(i+1)>\sigma_{\alpha}(i) \\
\alpha_{\sigma_{\alpha}(i+1)}>\alpha_{\sigma_{\alpha}(i)} \quad \text { if } \quad \sigma_{\alpha}(i+1)<\sigma_{\alpha}(i) .
\end{array}
$$

The modification of the Yang-Baxter equation (2.9i) enforces a particular rule for the handling of the integer valued parameter $l$ in the formation of the intertwining matrix $R^{\sigma}(l)$ (related to the permutation $\sigma$ ), which can be read off from the modified composition law

$$
\begin{aligned}
R^{\sigma \sigma_{i}}(l) & =R_{\sigma(i), \sigma(i+1)}\left(\tilde{l}_{i}\right) R^{\sigma}(l) ; \\
\tilde{l}_{i} & =l-\sigma_{\sigma(1)}-\ldots-\sigma_{\sigma(i-1)},
\end{aligned}
$$

where $\sigma_{i}$ is the transposition of $i, i+1$, and $\sigma$ an arbitrary permutation.

$R^{\sigma}(l)$ has the intertwining property

$$
R^{\sigma}(l) T_{0,1 \ldots N}(l)=T_{0, \sigma(1) \ldots \sigma(N)}(l) R^{\sigma}\left(l-\sigma_{0}\right) .
$$

The matrix $F_{1 \ldots N}(l)$ satisfies the factorizing equation

$$
R_{1 \ldots N}^{\sigma \quad}(l)=F_{\sigma(1 \ldots N)}^{-1}(l) F_{1 \ldots N}(l) .
$$

A proof of the latter equation can be found in [i]미].

The operators of the monodromy matrix $\left(\begin{array}{c}1 \\ 2\end{array} . \overline{1} \overline{0}\right)$ in the $F$ basis are obtained by using a recursion relation, which enables one to express the monodromy matrix for $N$ sites in terms of that for $N-1$ sites $[\overline{7} \overline{7}]$ :

$$
\begin{array}{r}
\tilde{T}_{0,1 \ldots N}(l)=\left(\begin{array}{cc}
\mathbb{I} & 0 \\
\tilde{C}_{2 \ldots N}^{1}(l) & \tilde{D}_{2 \ldots N}^{1}(l)
\end{array}\right)_{[1]} \times \\
\tilde{T}_{0,2 \ldots N}\left(l-\sigma_{1}\right) R_{01}(l) \times \\
\left(\begin{array}{cc}
\mathbb{I} & 0 \\
\tilde{C}_{2 \ldots N}^{1}\left(l-\sigma_{0}\right) & \tilde{D}_{2 \ldots N}^{1}\left(l-\sigma_{0}\right)
\end{array}\right)_{[1]}^{-1} .
\end{array}
$$

This relation can be solved recursively starting with the one site monodromy matrix which coincides with the Lax operator $L_{i}=R_{0 i}(2.8 i)$.

The solution of the recursion relation finally yields the following result for the operators of the monodromy matrix (we use a slight change in notation: $b(\lambda)=\frac{h(\lambda)}{h(\lambda+2 \eta)}$ and denote $\left.k=l-\sum_{i=1}^{N} \sigma_{i}\right)$ :

$$
\begin{aligned}
& \tilde{D}_{l}\left(\lambda_{0}\right)= \\
& \frac{h\left(\omega_{l+1}\right)}{h\left(\omega_{1+\frac{k+l-N}{2}}\right)} \otimes_{i=1}^{N}\left(\begin{array}{cc}
b\left(\lambda_{0 i}\right) & 0 \\
0 & 1
\end{array}\right)_{[i]} \text {; } \\
& \tilde{B}_{l}\left(\lambda_{0}\right)=\frac{h\left(\omega_{l+1}\right)}{h\left(\omega_{k}\right)} \sum_{i=1}^{N} c_{k-1}\left(\lambda_{0 i}\right) \sigma_{i}^{-} \times \\
& \otimes_{j \neq i}^{N}\left(\begin{array}{cc}
b\left(\lambda_{0 j}\right) & 0 \\
0 & b^{-1}\left(\lambda_{j i}\right)
\end{array}\right)_{[j]} ; \\
& \tilde{C}_{l}\left(\lambda_{0}\right)=\sum_{i=1}^{N} c_{l}^{\prime}\left(\lambda_{0 i}\right) \sigma_{i}^{+} \times \\
& \otimes_{j \neq i}^{N}\left(\begin{array}{cc}
b\left(\lambda_{0 j}\right) b^{-1}\left(\lambda_{i j}\right) & 0 \\
0 & 1
\end{array}\right)_{[j]} ;
\end{aligned}
$$




$$
\begin{aligned}
& \begin{array}{r}
\tilde{A}_{l}\left(\lambda_{0}\right)= \\
\frac{h\left(\omega_{\frac{k+l-N}{2}}\right)}{h\left(\omega_{k}\right)}\left\{\otimes_{i=1}^{N}\left(\begin{array}{cc}
1 & 0 \\
0 & b\left(\lambda_{i 0}\right)^{-1}
\end{array}\right)_{[i]}+\right.
\end{array} \\
& \sum_{i=1}^{N} \frac{c_{k-1}\left(\lambda_{0 i}\right) c_{l}^{\prime}\left(\lambda_{0 i}\right)}{b\left(\lambda_{0 i}\right)}\left(\begin{array}{ll}
0 & 0 \\
0 & 1
\end{array}\right)_{[i]} \times \\
& \otimes_{j \neq i}\left(\begin{array}{cc}
\frac{b\left(\lambda_{0 j}\right)}{b\left(\lambda_{i j}\right)} & 0 \\
0 & b\left(\lambda_{j i}\right)^{-1}
\end{array}\right)_{[j]} \\
& +\sum_{i \neq j}^{N} \frac{c_{k-1}\left(\lambda_{0 i}\right) c_{l}^{\prime}\left(\lambda_{0 j}\right)}{b\left(\lambda_{j}-\lambda_{k}\right)} \sigma_{-}^{i} \otimes \sigma_{+}^{j} \times \\
& \left.\otimes_{k \neq i, j}\left(\begin{array}{cc}
\frac{b\left(\lambda_{0 k}\right)}{b\left(\lambda_{j k}\right)} & 0 \\
0 & b\left(\lambda_{k i}\right)^{-1}
\end{array}\right)_{[k]}\right\},
\end{aligned}
$$

where $\lambda_{i j}=\lambda_{i}-\lambda_{j}$.

The above mentioned basis transformation (2.2.5) amounts to splitting the model into sectors with a fixed number of turned spins. To obtain the spectrum of the $X Y Z$ model one uses the operators $\tilde{B}_{l}(\lambda), \tilde{C}_{l}(\lambda)$ to construct eigenvectors in the form proposed by [i]] (there denoted by $B_{k, l}(\lambda)$ etc. $)$.

\section{Conclusion}

The form of the F-matrix, Eq. (3.2i) and the appearance of the monodromy matrix in the basis supplied by the F-matrix, Eq's (3.7i), , are completely analogous to what has been found in [1, [1] and $[6]$ for the rational and trigonometric models. The concrete expressions for $\tilde{A}, \tilde{B}, \tilde{C}, \tilde{D}$ are in particular manifestly symmetric with respect to exchanges of the local inhomogeneity parameters $\lambda_{i}$. The quasiparticle operators $\tilde{B}$ and $\tilde{C}$ are free from polarization effects due to non-local exchange terms.

The argument used in [6, [6] - borrowed from [i] concerning the identification of operators corresponding to different entries of the monodromy matrix relied on the $s l(n)$ symmetry of the rational model. It is not available for the trigonometric and elliptic model. The recursive procedure followed instead in the preceding section is equally applicable to the $X X X, X X Z$ and $X Y Z$ model.
It seems rather plausible in view of the formal similarities of the rational, trigonometric and elliptic models that some version of Sklyanin's functional Bethe ansatz should also be feasible in the latter case as has already been achieved for the $X Y Z$ Gaudin magnet in [i] $\left.{ }_{1}^{1}\right]$.

Acknowledgement: H.B. and R.P. acknowledge support of the Alexander von Humboldt Foundation. R.F. was supported by the TMR network contract FMRX-CT96-0012 of the European Commission.

\section{References}

[1] Maillet, J. M., Sanchez de Santos, J.: Drinfel'd twists and algebraic Bethe ansatz, qalg/9612012.

[2] Drinfel'd, V. G.: Quasi-Hopf algebras, Leningrad Math. J. 1, (1990), 1419.

[3] Terras, V.: Drinfel'd twists and functional Bethe ansatz, Lett. Math. Phys. 48, (1999), 263276, math-ph/9902009.

[4] Sklyanin, E. K.: Functional Bethe Ansatz, in: Integrable and Superintegrable Systems, B. Kupershmidt (ed.), World Scientific, Singapore, 1990, pp. 8-33.

[5] Baxter, R. J.: Eight vertex model in lattice statistics and one-dimmensional anisotropic Heisenberg chain I-III, Ann. Phys. (N.Y.) 76, (1973), 1-71.

[6] Albert, T.-D., Boos, H., Flume, R., Ruhlig, K.: Resolution of the nested hierarchy for rational sl(n) models, nlin-SI/0002027.

[7] Albert, T.-D., Boos, H., Flume, R., Poghossian, R. , Ruhlig, K.: The Drinfel'd twisted XYZ model, nlin-SI/0005023.

[8] Faddeev, L. D., Takhtadzhan, L. A.: The quantum version of the inverse problem and the Heisenberg XYZ model, Russ. Math. Surveys 34, (1979), 11-68.

[9] Babujian, H., Lima-Santos, A., Poghossian, R. H.: Knizhnik-Zamolodchikov-Bernard equations connected with the eight-vertex model, Int. Journ. Mod. Phys. A 14, (1999), 615-630, nlin-SI/9804015.

[10] Sklyanin, E. K., Takebe, T.: Algebraic Bethe ansatz for the XYZ Gaudin magnet, Phys. lett., A 219, (1996), 217-225, q-alg/9601028. 
[11] Gervais, J. L., Neveu, A.: Novel triangle relation and absence of tachyons in Liouville field theory, Nucl. Phys. B 238, (1984), 125.

[12] Felder, G.: Elliptic quantum groups, hepth/9412207. 